\title{
Rehabilitation of mutilated dentition associated with amelogenesis imperfecta
}

Bajracharya $\mathrm{A}^{1}$, Karn $\mathrm{SL}^{2}$, Shrestha $\mathrm{A}^{3}$

${ }^{1}$ Consultant Prosthodontist, Himal Dental Hospital, Kathmandu, Nepal.

${ }^{2,3}$ Assistant Professor, Peoples Dental College and Hospital, Kathmandu, Nepal

\begin{abstract}
This clinical case report describes the oral rehabilitation of a young male with hypoplastic Amelogenesis Imperfecta with generalized hypoplastic and pitted teeth. All the teeth showed yellowish brown discoloration with rough irregular crowns. Patient was a young male who dissatisfied with his appearance and his condition degraded his confidence and psychology. Numerous treatment modalities have been described for rehabilitation of AI patient but the limitations in the treatment exists and the application of techniques are not universal The main objective of this selected treatment was to enhance the esthetics, restore masticatory function and eliminate the teeth sensitivity. These treatment objectives were successfully met by sequential approach. Treatment not only restored function and esthetic, but also showed a positive psychological impact and thereby improved perceived quality of life.
\end{abstract}

Key words: Amelogenesis imperfecta; Mutilated dentition; Fixed prosthodontics; Full mouth rehabilitation

\section{INTRODUCTION}

$\mathrm{A}$ melogenesis imperfecta (AI) is defined as a group of hereditary developmental defects of the dental enamel affecting both primary and permanent dentition. ${ }^{1}$ It has variable prevalence from $1 / 700$ to $1 / 14,000$ in United States. ${ }^{2}$ AI is caused by mutations in genes that control amelogenesis. $^{3}$ AI may be inherited in an Xlinked manner or by autosomal dominant, autosomal recessive, or sporadic inheritance patterns. $^{4,5}$ Mutation or changed expression of 13 genes have been identified as causative agents for AI, including AMELX, ENAM, MMP20, KLK4, FAM83H, WDR72, FAM20A,

\section{Conflict of Interest: No}

\section{*Corresponding Author}

Dr. Ayusha Bajracharya, Consultant Prosthodontist, Himal Dental Hospital, Kathmandu, Nepal

E-mail:ayushabjr@gmail.com
SLC24A4, DLX3, AMBN, LAMB3, ITGB6, and C4orf26. ${ }^{6}$ The most common type of AI occurs as a result of the autosomal dominant form of transmittance. ${ }^{7}$ This anomaly exists independent of any related systemic diseases. ${ }^{7,8,9}$ There are various classification systems proposed for the different types of AI. Generally, $\mathrm{AI}$ is classified into three types on the basis of clinical and radiographic findings - hypoplastic, hypomineralization, and hypomaturation ${ }^{7,10}$, ${ }^{11}$ In cases of hypoplastic AI, the thickness of the enamel layer is reduced, and enamel tissues can be radiographically distinguished from dentine. $^{12}$ In hypomaturated $\mathrm{AI}$, the enamel layer features a physiological thickness, but appears mottled and softer than sound enamel; radiopacity is similar to dentine. The thickness of the enamel layers is physiological in cases of hypocalcified AI, yet due to the impaired calcification the enamel surfaces are subject to increased wear. Radiographically, enamel appears less radiopaque than dentine. ${ }^{13}$ 
The primary clinical problems of AI include poor dental esthetics, dental sensitivity, and loss of vertical dimension. ${ }^{14}$ The enamel is abnormally thin, soft, fragile, pitted and/or badly discolored with poor function and aesthetics, causing patients problems such as early tooth loss, severe embarrassment, eating difficulties, and pain. Moreover, the AI patient has an increased probability for having dental caries and plaque accumulation. AI also has been associated with impacted teeth and anomalies in tooth eruption, congenitally missing teeth, pulp calcification, root and coronal resorption, hypercementosis, root malformation, and taurodontism. ${ }^{13-17}$ Restorations are important to restore esthetic, function and psychology of the patient.

The treatment planning for patients with $\mathrm{AI}$ is related to many factors, including the age of the patient, the type and severity of the disorder, intraoral conditions, and the socioeconomic status of the patient. ${ }^{15}$ There are many options proposed for the treatment of AI-affected teeth, including simple microabrasion, ${ }^{16}$ composite veneers, ${ }^{17}$ porcelain laminate veneers, onlays, ${ }^{11}$, 18 gold or stainless-steel crowns, ${ }^{18-21}$ metal ceramic crowns, and all-ceramic crowns. ${ }^{11}$, ${ }^{18}$ Both direct and indirect treatment options are regularly suggested for the restorative rehabilitation of patients with AI, however, it is better to postpone the rehabilitation in adolescents with indirect restorations for preventing potential endodontic complications. The longevity of dental restorations in patients with AI correlates with the severity of AI. The survival rates are higher in patients with hypoplastic types of AI than in patients with hypomaturated or hypocalcified types of AI. ${ }^{22}$

This clinical report is of 18 years old young male with mutilated natural dentition associated with Amelogenesis Imperfecta in which function and esthetic was restored using full veneer crowns in anterior and posterior teeth.

\section{Methods}

18 years old male patient visited the Department of Prosthodontics with complain of severely discolored upper and lower dentition. Patient was dissatisfied with his appearance but had no complaint of pain or sensitivity in the teeth. Family history revealed that his brother also had similar dental condition. There was no history of systemic disease, nutritional deficiency, or drug treatments that may have affected dentition during development. Patient had no past dental history.

On examination, the patient's anterior and posterior teeth were hypoplastic and pitted (Figure 1). All teeth showed yellowish brown discoloration with rough irregular crowns. There was attrition in the upper posterior with moderate loss of tooth structure whereas the lower posteriors were normal in anatomy. Oral hygiene was poor, with evidence of gingivitis due to severe plaque accumulation. There was not much loss of vertical dimension. No teeth were carious. The maxillary and mandibular teeth were well aligned with overjet $0.5 \mathrm{~mm}$ and overbite as $2 \mathrm{~mm}$ (Figure 2) Patient had group function occlusion on left and right side. Smile analysis showed high lip line, parallel smile line, straight upper lip curvature and symmetrical smile.

Diagnostic impressions were made with irreversible hydrocolloid impression material and poured in type III dental stone to obtain diagnostic casts. Diagnostic casts were mounted and OPG (Figure 3) was taken. Using mounted diagnostic models, along with clinical and radiographic findings, the treatment plan was formulated with the aim to improve esthetics and restore masticatory function.

The patient was informed about the diagnosis and treatment plan with its objectives and possible complications and all other treatment 
alternatives including the differences in costs, the levels of tooth structure removal, the expected clinical longevity, the time period necessary to conclude the treatment, and the possible esthetic result was given to the patient.

The treatment included oral prophylaxis followed by full mouth rehabilitation using full veneer all ceramic lithium disilicate crowns in anterior teeth followed by porcelain fused metal crown in the posterior teeth due to financial restrictions. Since there was no carious involvement and the radiographic evaluation showed no involvement of the pulp, none of the teeth were planned for root canal treatment.

Patient consent was taken. Following dental prophylaxis and oral hygiene instructions, the patient was placed on a $0.12 \%$ chlorohexidine oral rinse, twice daily.

Patient had 3-4 mm of interocclusal distance thus the patient's existing vertical dimension was not much altered. Diagnostic mockup (Figure 4) was done to restore the lost cusp fossa relation in the occlusal surface of the posterior teeth followed by anterior to restore desired esthetic with adequate overjet and overbite. This was also used to fabricate provisional restoration.

Upper and lower posterior teeth were prepared, first on one side then on the other side in next appointment. During tooth preparation it was noticed that on removal of the pitted, discoloured

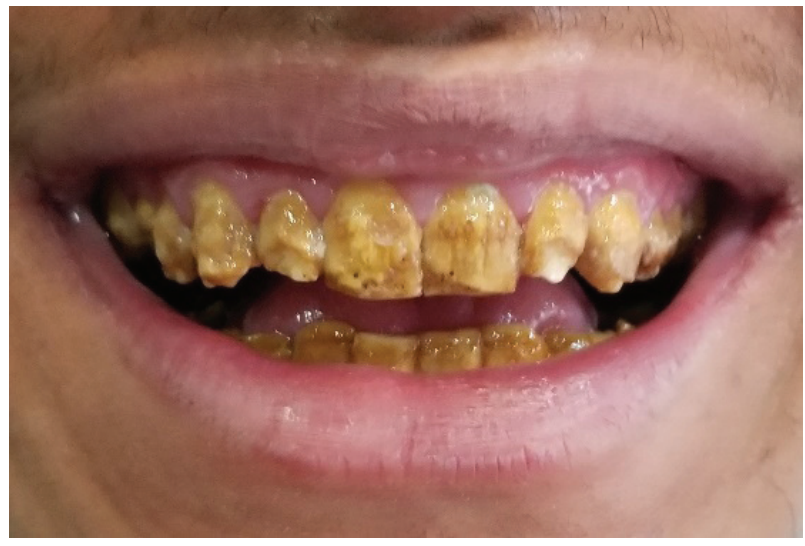

Figure 1: Hypoplastic, discolored, pitted dentition tooth structure the dentine structure was sound and healthy as of a normal dentition. On the day of tooth preparation temporary crown was prepared using direct method using the model index and bisacryl on patients mouth. Due to the multiunit preparation, and properties of bisacryl to be brittle, poor color stability and poor stain resistance bisacryl was replaced by acrylic provisional (Figure 5) in later appointment. This temporary restored the lost height of the posterior as well as guided adequate space for the crowning of the anterior teeth (Figure 6).

Patient was recalled after 3 weeks. As there was minimum rise in the vertical dimension patient quickly adapted to the new occlusion. There was no history of discomfort, nor any muscle or TMJ pain with the provisional. Next the anterior teeth were prepared and provisional was placed (Figure 7).

Patient was recalled after 2 weeks for final impression. Teeth were restored with porcelain fused to metal crown in upper and lower posterior teeth. Occlusal correction was done in both centric and eccentric movements. On later appointment, lithium disilicate full veneer crown was placed in anterior teeth using self adhesive resin cement (Figure 8). Canine guided occlusion was given to the patient. Patients' functional and esthetic rehabilitation was done (Figure 9). Patient was given oral hygiene instruction and advised to follow up every 6 months.

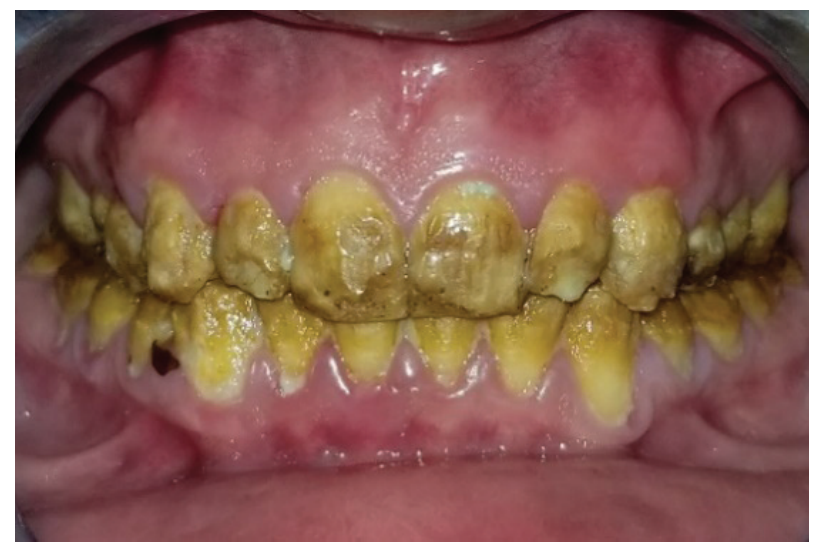

Figure 2: Overjet and overbite 


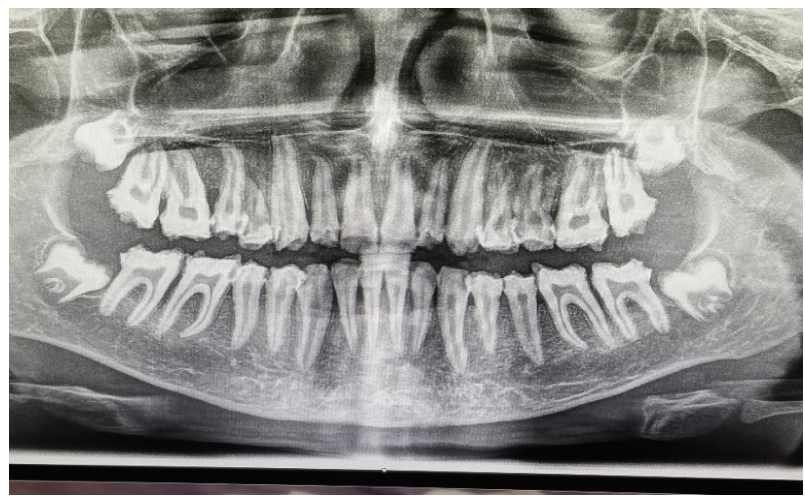

Figure 3: $O P G$

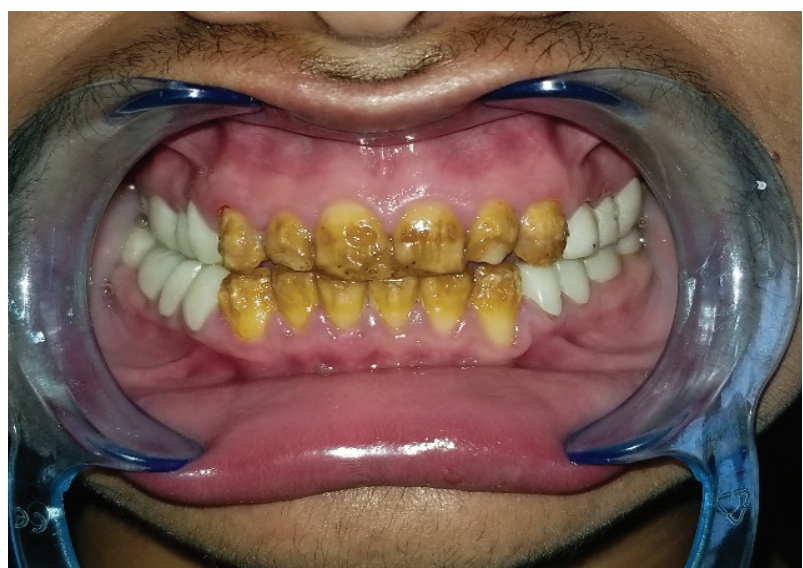

Figure 5: Posterior provisional restoration

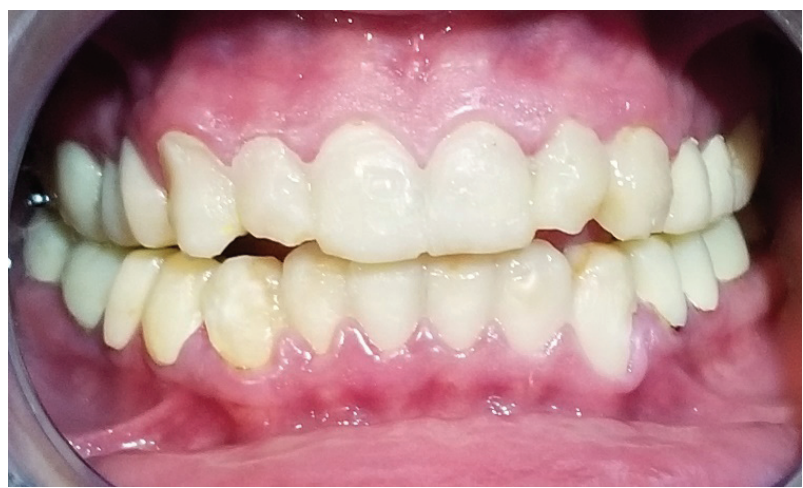

Figure 7: Complete provisionalization

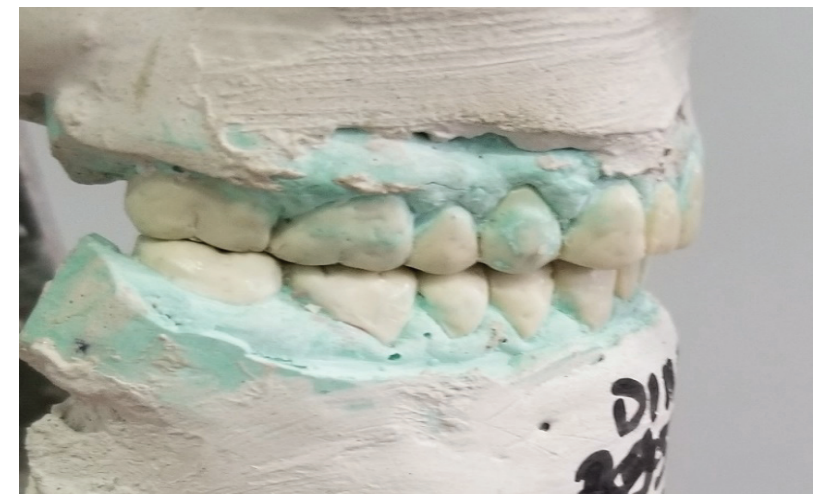

Figure 4: Mock up

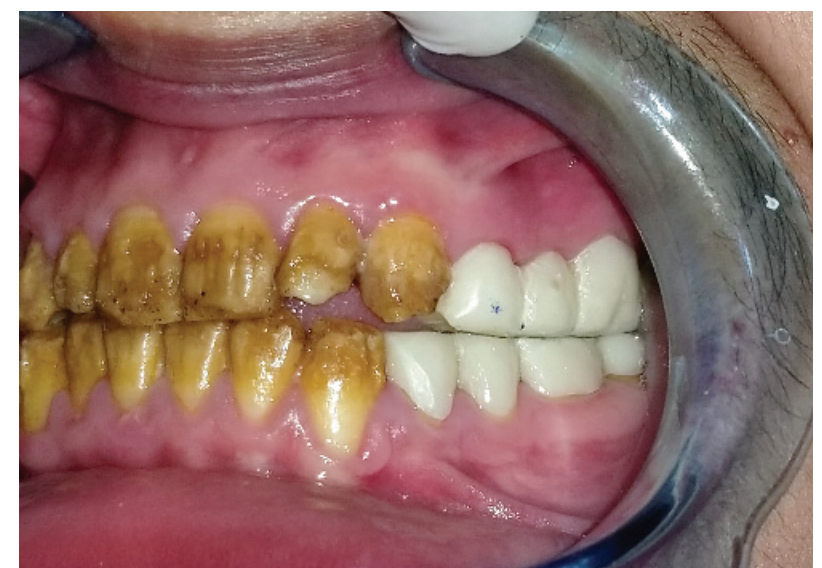

Figure 6: Raised vertical dimension

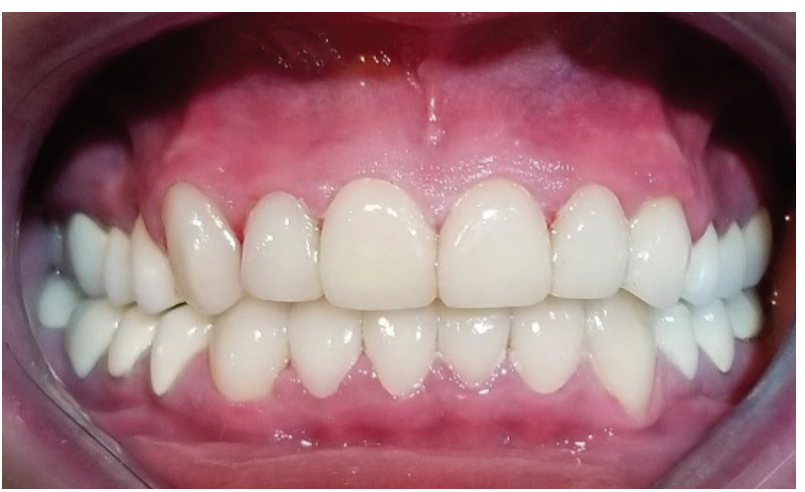

Figure 8: Lithium disilicate and PFM crowns

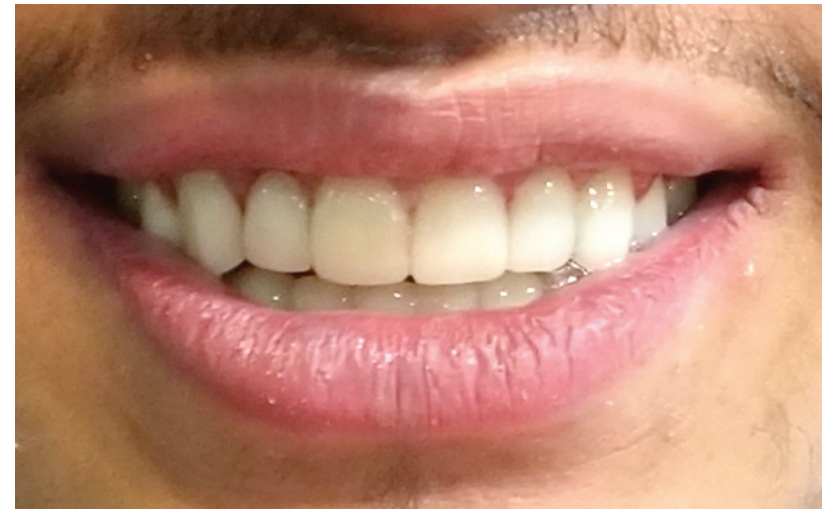

Figure 9: Patient smile 


\section{Discussion}

Timely diagnosis and individual approach is important for adequate treatment of patients with Amelogenesis imperfecta. Numerous treatment modalities have been described for rehabilitation of AI patient but the limitations in the treatment exists and the application of techniques are not universal. ${ }^{23}$ The treatment for patients with amelogenesis imperfecta is related to many factors including the age of patients, the socio-economic status, the type and severity of the disorder, its intra oral manifestation, aesthetic and functional demands. Treatment plan should have common goals i.e., functional, aesthetic and longevity of restoration, yet the approaches being slightly different. Adhesive restorative techniques, over-dentures, porcelain fused-to metal crowns, fixed partial dentures, full porcelain crowns, and inlay/onlay restorations can all be used for the prosthetic rehabilitation of AI patients depending on the case..$^{23-25}$

The main reason for failure of direct restorations in patients with $\mathrm{AI}$ is fracture of the restoration or of the tooth. The reduced longevity of adhesive restorations can be explained by the reduced amount and quality of the existing enamel. Treatment with direct composite restorations is mostly performed as a temporary therapy to gain some time and to perform the definitive restoration in an older stage of life or due to financial reasons. ${ }^{26,27}$

Indirect restoration has been commonly employed for the rehabilitation of AI. Regardless of conventional or adhesive cementation protocols, loss of retention of indirect restorations in patients with $\mathrm{AI}$ is infrequent due to almost complete removal of irregular enamel and additional mechanical retention. ${ }^{28}$ Laboratory studies addressing the adhesive bond strength to AI affected enamel and dentin identified significantly higher adhesive bond strength to dentin in comparison to enamel from
AI-affected patients, although values were still significantly lower than for dentin from healthy teeth. ${ }^{28}$ These observations suggest that in cases where the irregular enamel layers are removed, indirect restorations might be effectively luted with adhesive cements in AI-affected teeth.

Newer, more aesthetic materials for full veneer restorations like lithium disilicate offer a further clinically relevant advantage as these materials require a reduced reduction of dental hard tissues during preparation. Lithium disilicate all-ceramic crowns require only a thickness of approximately $0.8 \mathrm{~mm}$ in the cervical region, and an occlusal reduction of only $1.5-2.0 \mathrm{~mm}$ is sufficient. ${ }^{29}$ This material allow a sufficient preparation of the affected teeth without an increased risk of iatrogenic irritation of the pulp tissue offering a better choice of treatment.

In this case since the patient was young and wanted a definite restoration so full veneer crown was planned. All ceramic lithium disilicate full veneer crown was placed in the esthetic region i.e upper and lower anterior teeth whereas porcelain fused to metal full veneer crown was planned for the posteriors.

\section{Conclusions}

This clinical report described the oral rehabilitation of a young male patient affected by Amelogenesis Imperfecta. Although several treatment options were presented as part of the optimum treatment plan to achieve the best result but the patient wanted definitive treatment with limited finance. Metal-ceramic full veneer crowns were placed on the posterior teeth and all ceramic lithium disilicate crowns were placed on the maxillary and mandibular anterior teeth to improve the occlusion and esthetics.

\section{References}

1. Coley-Smith A, Brown C. Case report: radical management of an adolescent with amelogenesis imperfecta. Dental update 1996;23(10):434-35. 
2. Crawford PJ, Aldred M, Bloch-Zupan A. Amelogenesis imperfecta. Orphanet journal of rare diseases 2007;2(1):1-11.

3. Aldred MJ SR, Crawford PJ (). . Amelogenesis imperfecta: a classification, catalogue for the 21 st century. Oral Dis 2003;9(1):19-23.

4. subcommittee AAoPDCAC-PT. American Academy on Pediatric Dentistry Council on Clinical Affairs: Guideline on pulp therapy for primary and young permanent teeth. Pediatr. Dent. 2009;30(7):170-74.

5. Canger EM, Celenk P, Yenisey M, Odyakmaz SZ. Amelogenesis imperfecta, hypoplastic type associated with some dental abnormalities: a case report. Brazilian dental journal 2010;21(2):170-74.

6. Hu JC-C, Chan H-C, Simmer SG, et al. Amelogenesis imperfecta in two families with defined AMELX deletions in ARHGAP6. PloS one 2012;7(12):e52052.

7. Bsoul SA, Flint DJ, Terezhalmy GT, Moore WS. Amelogenesis imperfecta. Quintessence international 2004;35(4):338-39.

8. Neville BW, Damm DD, Allen CM, Chi AC. Oral and maxillofacial pathology: Elsevier Health Sciences; 2015.

9. Aldred M, Savarirayan R, Crawford P. Amelogenesis imperfecta: a classification and catalogue for the 21 st century. Oral diseases 2003;9(1):19-23.

10. Soares CJ, Fonseca RB, Martins LRM, Giannini M. Esthetic rehabilitation of anterior teeth affected by enamel hypoplasia: a case report. Journal of esthetic and restorative dentistry 2002;14(6):340-48.

11. Nel JC, Pretorius JA, Weber A, Marais J. Restoring function and esthetics in a patient with amelogenesis imperfecta. International Journal of Periodontics \& Restorative Dentistry 1997;17(5).

12. Witkop Jr C. Amelogenesis imperfecta, dentinogenesis imperfecta and dentin dysplasia revisited: problems in classification. Journal of Oral Pathology \& Medicine 1988;17(9-10):54753.

13. Gadhia K, McDonald S, Arkutu N, Malik K. Amelogenesis imperfecta: an introduction. British dental journal 2012;212(8):377-79.
14. Robinson FG, Haubenreich JE. Oral rehabilitation of a young adult with hypoplastic amelogenesis imperfecta: a clinical report. The Journal of prosthetic dentistry 2006;95(1):1013.

15. Sari T, Usumez A. Restoring function and esthetics in a patient with amelogenesis imperfecta: a clinical report. The Journal of prosthetic dentistry 2003;90(6):522-25.

16. Ashkenazi M, Sarnat H. Microabrasion of teeth with discoloration resembling hypomaturation enamel defects: four-year follow up. Journal of Clinical Pediatric Dentistry 2001;25(1):29-34.

17. Sabatini C, GUZMÁN-ARMSTRONG S. A conservative treatment for amelogenesis imperfecta with direct resin composite restorations: a case report. Journal of Esthetic and Restorative Dentistry 2009;21(3):161-69.

18. Yip H-K, Smales RJ. Oral rehabilitation of young adults with amelogenesis imperfecta. International Journal of Prosthodontics 2003;16(4).

19. Bouvier D, Duprez J-P, Pirel C, Vincent B. Amelogenesis imperfecta - a prosthetic rehabilitation: a clinical report. Journal of Prosthetic Dentistry 1999;82(2):130-31.

20. Quinonez R, Hoover R, Wright J. Transitional anterior esthetic restorations for patients with enamel defects. Pediatric dentistry 2000;22(1):65-67.

21. Rosenblum SH. Restorative and orthodontic treatment of an adolescent patient with Amelogenesis Imperfects. Pediatric Dentistry 1999;21:289-92.

22. Lundgren GP, Dahllöf G. Outcome of restorative treatment in young patients with amelogenesis imperfecta. a cross-sectional, retrospective study. Journal of dentistry 2014;42(11):138289.

23. Kim Li RW. Adhesive solutions: report of a case using multiple adhesive techniques in the management of enamel hypoplasia. Dental update 1999;26(7):277-87.

24. Gokce K, Canpolat C, Ozel E. Restoring function and esthetics in a patient with amelogenesis imperfecta: a case report. J Contemp Dent Pract 2007;8(4):95-101. 
25. Williams WP, Becker LH. Amelogenesis imperfecta: Functional and esthetic restoration of a severely compromised dentition. Quintessence International 2000;31(6).

26. Horowitz RA, Gautam D, Karol S, Kumari B. Periodontal management and restoration of an amelogenesis imperfecta patient: a case report. Compendium of continuing education in dentistry (Jamesburg, NJ: 1995) 2014;35(2):e611.

27. Mittal S, Tewari S, Goel R. Esthetic and functional rehabilitation of mutilated dentition and loss of vertical dimension due to amelogenesis imperfecta. Indian journal of dentistry 2014;5(2):102.

28. Pousette Lundgren G, Morling Vestlund G, Trulsson M, DahllöfG. A randomized controlled trial of crown therapy in young individuals with amelogenesis imperfecta. Journal of dental research 2015;94(8):1041-47.

29. Gehrt M, Wolfart S, Rafai N, Reich S, Edelhoff D. Clinical results of lithium-disilicate crowns after up to 9 years of service. Clinical oral investigations 2013;17(1):275-84. 